\title{
Diagnosis and Management of Genetic Iron Overload Disorders
}

\author{
William C. Palmer, MD, FACP ${ }^{\top}$, Prakash Vishnu, MBBS, FACP', William Sanchez, MD, FAASLD ${ }^{3}$, \\ Bashar Aqel, MD', Doug Riegert-Johnson, MD' ${ }^{7}$, Leigh Ann Kenda Seaman, RDN, LD/N ${ }^{5}$, \\ Andrew W. Bowman, MD, PhD $D^{6}$, and Candido E. Rivera, MD, FACP2
}

\begin{abstract}
'Department of Gastroenterology and Hepatology, Mayo Clinic, Jacksonville, FL, USA; ${ }^{2}$ Department of Hematology/Oncology, Mayo Clinic, Jacksonville, FL, USA; ${ }^{3}$ Department of Gastroenterology and Hepatology, Mayo Clinic, Rochester, MN, USA; ${ }^{4}$ Department of Gastroenterology and Hepatology, Mayo Clinic, Scottsdale, AZ, USA; 5 Department of Nutrition, Mayo Clinic, Jacksonville, FL, USA; ${ }^{6}$ Department of Radiology, Mayo Clinic, Jacksonville, FL, USA.
\end{abstract}

Iron overload disorders lead to excess iron deposition in the body, which can occur as a result of genetic or secondary causes. Genetic iron overload, referred to as hereditary hemochromatosis, may present as a common autosomal recessive mutation or as one of several uncommon mutations. Secondary iron overload may result from frequent blood transfusions, exogenous iron intake, or certain hematological diseases such as dyserythropoietic syndrome or chronic hemolytic anemia. Iron overload may be asymptomatic, or may present with significant diseases of the liver, heart, endocrine glands, joints, or other organs. If treated appropriately prior to end-organ damage, life expectancy has been shown to be similar compared to matched populations. Alongside clinical assessment, diagnostic studies involve blood tests, imaging, and in some cases liver biopsy. The mainstay of therapy is periodic phlebotomy, although oral chelation is an option for selected patients.

KEY WORDS: hemochromatosis; iron overload.

J Gen Intern Med 33(12):2230-6

DOI: $10.1007 / \mathrm{s} 11606-018-4669-2$

(c) Society of General Internal Medicine 2018

\section{INTRODUCTION}

Disorders of iron metabolism can result from genetic abnormalities or secondary disturbances that result from excessive blood transfusion, dyserythropoietic syndrome, chronic hemolysis, or iron supplementation, and manifest accordingly as primary or secondary iron overload state (IOS). IOS, a potentially serious condition, is often overlooked as the symptoms are non-specific and often develop gradually. IOS can manifest with heterogeneous clinical features: skin discoloration, arthritis, abdominal pain, infertility, impotence, cardiac arrhythmia, heart failure, new-onset or difficult-to-control diabetes mellitus, and elevation in liver enzymes. Hereditary hemochromatosis (HH), the most common genetic iron overload disorder, can also lead to development of severe complications such as cirrhosis of the liver

Received November 9, 2017

Revised May 23, 2018

Accepted September 7, 2018

Published online September 17, 2018 and hepatocellular carcinoma. ${ }^{1}$ Manifestations of iron overload in $\mathrm{HH}$ are related to mutations of certain genes involved in iron metabolism that causes increased intestinal iron absorption. The primary defect in $H F E$ gene causing $\mathrm{HH}$ was first described in $1996 .^{2}$ However, over the past two decades, mutations of other genes coding for iron regulatory proteins such as hemojuvelin (HJV), hepcidin (HAMP), transferrin receptor 2 (TFR2), and ferroportin (SLC40A1) have all been implicated in causing genetic IOS.

The presence of one or more of the above signs and symptoms and/or family history of IOS should raise suspicion for the presence of $\mathrm{HH}$ and to perform the necessary testing. Once IOS is diagnosed, the treatment options are relatively forthright in most patients. Nonetheless, some untreated individuals can develop life-threatening organ damage, and hence it is important to identify iron overload before such organ damage occurs.

This article, intended to be used by physicians and health care providers, outlines the data-supported approach to the diagnostic, therapeutic, and preventative aspects of care in patients with genetic IOS, as well as provides monitoring recommendations and best-practice suggestions from a highvolume iron disorder referral center.

\section{GENETICS AND RISK FACTORS}

Genetic IOS, referred to as $\mathrm{HH}$, can be divided into two categories: $H F E$-associated and non- $H F E$-associated. The majority of genetic mutations directly or indirectly affect hepcidin, a 25-amino acid peptide produced by hepatocytes involved in a feedback loop which controls intestinal iron absorption.

$H F E$-associated $\mathrm{HH}$, also known as type $\mathrm{I} \mathrm{HH}$, most commonly presents as a compound homozygote $(80 \%)$ of the $\mathrm{C} 282 \mathrm{Y}$ mutation $(\mathrm{C} 282 \mathrm{Y} / \mathrm{C} 282 \mathrm{Y})$, with a $50-90 \%$ penetrance of iron overload. HFE gene mutations are common in those of northern European ancestry, with occurrence in $1 / 250$ people with this heritage. ${ }^{3}$ The cause for this limited penetrance is not clear and is the bases for limiting general population screening. ${ }^{4}$ Two other less common $H F E$-associated $\mathrm{HH}$ genotypes are compound 
heterozygotes of $\mathrm{C} 282 \mathrm{Y} / \mathrm{H} 63 \mathrm{D}$ or $\mathrm{C} 282 \mathrm{Y} / \mathrm{S} 65 \mathrm{C}$, with both of these genotypes having approximately $5 \%$ penetrance of iron overload. ${ }^{5}$ Compound homozygote of H63D (H63D/H63D) may predispose to iron overload, but evidence is sparse and likely represents a milder phenotype. Carrier states (wt/C282Y, wt/H63D, wt/S65C) are not classically associated with $H F E$-associated $\mathrm{HH}$, but recent evidence suggests that they may indeed predispose to IOS or other conditions. ${ }^{6-8}$

Non- $H F E$-associated $\mathrm{HH}$ represents an uncommon spectrum of genetic mutations predisposing to iron overload (Table 1). These identified mutations are thought to be responsible for most non-HFE-associated $\mathrm{HH}$ cases, but other rarer gene mutations are likely yet to be discovered. ${ }^{1,9}$ These genetic conditions include those associated with mutations of hemojuvelin (HJV), hepcidin antimicrobial peptide (HAMP), transferrin receptor 2 (TfR2) mutations, and ferroportin (SLC40A1). Ferroportin, unlike the other mutations listed above, is inherited in an autosomal dominant pattern. These non$H F E$-associated conditions should be considered when gene testing for $H F E$ is negative, yet iron overload is clinically present.

Next-generation sequencing may provide further tools for those with more uncommon mutations predisposing to iron overload. ${ }^{10}$ Whole genome sequencing can provide more detailed data to associate mutations with iron overload. ${ }^{11}$
Secondary iron overload is related to frequent blood transfusions, exogenous iron intake, or certain hematological diseases such as sickle cell anemia or thalassemia. A combination of an $H F E$ carrier state with a secondary cause of iron overload may predispose to more significant disease. Exogenous iron supplementation can rarely cause significant iron overload and requires substantial iron intake. Parenteral iron infusions may cause laboratory and imaging changes reflective of increased systemic iron for a brief period following administration and should be interpreted with caution. African iron overload (Bantu siderosis) is a secondary IOS first observed in subSaharan Africa and primarily linked to iron consumption from traditional homemade beer with high iron content. ${ }^{12}$ Given the rarity of this condition, the role of ferroportin polymorphisms (SLC4OA1) in increasing the risk of significant iron overload in this population has been suggested. ${ }^{13,14}$

\section{SIGNS AND SYMPTOMS}

Most iron overload patients are asymptomatic at diagnosed. Patients may present with a variety of symptoms in the later stages of the disease.

Constitutional Symptoms. Fatigue and/or weakness can sometimes be attributed to iron overload and does tend to respond to iron removal. Importantly however, nonspecific

Table 1 Genetic and Clinical Profiles of Hereditary Hemochromatosis

\begin{tabular}{|c|c|c|c|c|c|c|}
\hline TYPE & GENE & FREQUENCY & INHERITANCE & IRON INDEX & CHARACTERISTICS & MECHANISM \\
\hline $\begin{array}{l}\text { Type 1: } \\
\text { Classic HH }\end{array}$ & HFE & Common & $\begin{array}{l}\text { Autosomal } \\
\text { recessive }\end{array}$ & $\begin{array}{c}\uparrow \text { Transferrin Sat } \\
\uparrow \text { Ferritin }\end{array}$ & $\begin{array}{l}\text { Onset } 4-5^{\text {th }} \text { decade, } \\
\text { parenchymal iron }\end{array}$ & $\downarrow$ Hepcidin \\
\hline $\begin{array}{l}\text { Type 2a: } \\
\text { Juvenile } 2 A\end{array}$ & $\begin{array}{l}\text { HV } \\
(1 q 21)\end{array}$ & Rare & $\begin{array}{l}\text { Autosomal } \\
\text { recessive }\end{array}$ & $\begin{array}{c}\uparrow \text { Transferrin Sat } \\
\uparrow \text { Ferritin }\end{array}$ & $\begin{array}{l}2^{\text {nd }} \text { decade, } \\
\text { hypogonadism, } \\
\text { cardiomyopathy }\end{array}$ & $\begin{array}{l}\downarrow \text { HAMP } \\
\text { activation } \\
\downarrow \text { Hepcidin }\end{array}$ \\
\hline $\begin{array}{l}\text { Type } 2 \mathrm{~b} \text { : } \\
\text { Juvenile } 2 \mathrm{~B}\end{array}$ & $\begin{array}{l}\text { HAMP } \\
(19 q 31)\end{array}$ & Rare & $\begin{array}{l}\text { Autosomal } \\
\text { recessive }\end{array}$ & $\begin{array}{c}\uparrow \text { Transferrin Sat } \\
\uparrow \text { Ferritin }\end{array}$ & $\begin{array}{l}3^{\text {rd }} \text { decade, } \\
\text { hypogonadism, } \\
\text { cardiomyopathy }\end{array}$ & $\begin{array}{l}\downarrow \text { Absent } \\
\text { Hepcidin }\end{array}$ \\
\hline $\begin{array}{c}\text { Type 3: } \\
\text { Transferrin } \\
\text { Receptor } 2 \\
\text { Deficiency (TfR2) }\end{array}$ & $\begin{array}{c}\text { TfR2 } \\
(7 q 22)\end{array}$ & Very Rare & $\begin{array}{l}\text { Autosomal } \\
\text { recessive }\end{array}$ & $\begin{array}{c}\uparrow \text { Transferrin Sat } \\
\uparrow \text { Ferritin }\end{array}$ & $\begin{array}{l}\text { Similar to Classic } \\
\mathrm{HH} \text {, onset } 2-4^{\text {th }} \\
\text { decade of life }\end{array}$ & $\downarrow$ Hepcidin \\
\hline $\begin{array}{l}\text { Type 4: } \\
\text { Ferroportin } \\
\text { Disease }\end{array}$ & $\begin{array}{c}\text { FPN } \\
\text { (SLC40A1) }\end{array}$ & Rare & $\begin{array}{l}\text { Autosomal } \\
\text { dominant }\end{array}$ & $\begin{array}{c}\uparrow \text { Transferrin Sat } \\
\uparrow \text { Ferritin }\end{array}$ & $\begin{array}{l}\text { Anemia, } \\
\text { reticuloendothelial } \\
\text { iron }\end{array}$ & $\uparrow$ Hepcidin \\
\hline
\end{tabular}


constitutional symptoms associated with an elevated ferritin can also be associated with a variety of other etiologies including autoimmune, metabolic, or infectious diseases. Therefore, confirming the presence of true iron overload in this setting is paramount. Free heme and hematin have been implicated as organotoxic elements in several studies, citing vascular inflammation and cerebral injury. ${ }^{15,16}$ However, these toxicities seem to be related to severe hemolysis and have not been directly linked to genetic IOS.

Musculoskeletal. Hemochromatosis-related arthritis typically present in the small joints, with the classic presentation of "iron fist" in the second and third metacarpal joints. Improvement in arthritis after phlebotomy is minimal. Population-based data from the National Inpatient Sample (NIS) suggests HH is also associated with increased odds of large joint replacement surgery compared to the general population, specifically hip replacement. ${ }^{17}$

Liver. Liver cirrhosis can occur after years of hepatic iron overload and tends to progress faster with concomitant liver diseases such as viral hepatitis, alcohol misuse, or nonalcoholic steatohepatitis. Therefore, screening and management of other causes of chronic liver injury are vital.

Cardiac. Restrictive heart failure due to dense iron deposition into the myocardium can present as a spectrum ranging from asymptomatic EKG conduction abnormities to debilitating heart failure. Diagnosis can typically be made with a cardiac magnetic resonance imaging (MRI) as detailed below, but in some cases, myocardial biopsy with iron quantification may be necessary. Classic echocardiogram findings reflect restrictive cardiomyopathy with ventricular strain.

Endocrine. Diabetes mellitus related to iron infiltration into the endocrine pancreatic tissue can present in the later stages of the disease and is sometimes referred to as "bronze diabetes" associated with yellow discoloration of the skin. Importantly, most patients with diabetes with elevated ferritin levels do not have clinical iron overload, but in fact have hyperferritinemia secondary to underlying inflammation. While iron deposition in the pancreas can be identified using MRI, non-invasive iron quantification MRI sequences (described below) currently are not as helpful or as well-established for the pancreas as they are for the liver and heart. Pituitary disturbance and loss of libido function present as iron deposition occurs in the gland, with predominant iron deposition in the anterior lobe of the pituitary gland. It can be quantified using brain MRI and is treated with hormone replacements. The most common hormones affected are gonadotropins, specifically testosterone. ${ }^{18}$ Growth hormone deficiency with anterior pituitary iron deposition has been reported. ${ }^{19}$ Antidiuretic hormone abnormalities have not been identified, explained by the classic sparing of the posterior pituitary lobe.

Malignancy. Liver cancer, specifically hepatocellular carcinoma, can present commonly in cirrhotic patients with iron overload. Patients with cirrhosis have an estimated 20- to 200-fold increased risk of hepatocellular carcinoma. Unfortunately, once $\mathrm{HH}$ patients develop cirrhosis, the risk for HCC does not decrease with phlebotomy. Large population study data suggest a potential connection with other malignancies including breast cancer and colon cancer in those with $H F E$ gene mutations. ${ }^{6,20}$ Further study in this area is needed.

\section{INITIAL ASSESSMENT AND DIAGNOSTICS}

Initial assessment (Fig. 1) of symptomatic patients should include a thorough history and physical, along with fasting serum testing for ferritin, total iron-binding capacity (TIBC), transferrin saturation, iron levels, and metabolic panel including hepatic enzymes (alanine aminotransferase and aspartate aminotransferase).

Isolated elevation in ferritin or TIBC can be caused by any inflammatory state and various other factors in addition to iron overload; further evaluation for patients who have an isolated elevation may include testing to identify or rule out particularly alcohol-related or non-alcoholic liver disease. Other conditions to consider include hemolysis, ineffective erythropoiesis, a systemic inflammatory disease, indolent infection, or a malignancy. Elevated ferritin levels in patients who consume alcohol should be rechecked after 3 months of abstinence. Elevated ferritin levels in patients who have features of the metabolic syndrome should be rechecked after 3 months of improvement in features of metabolic syndrome (diabetes, cholesterol, obesity).

In certain cases, iron overload can be estimated by imaging. Liver biopsy should be reserved for scenarios where the diagnosis is questionable, fibrosis quantification is needed and not technically feasible through noninvasive means, or a second overlapping liver disease is being considered.

\section{SCREENING}

Fasting ferritin and transferrin saturation levels should be obtained in patients with chronic elevation in liver enzymes, or in those with unexplained signs and symptoms outlined previously. Targeted population-based screening for asymptomatic men of northern European ancestry can also be considered. ${ }^{21}$ Ferritin, being an acute-phase reactant can be elevated in response to processes that do not correlate with iron status, including inflammation, chronic disease, malignancy, and infection. Soluble transferrin 


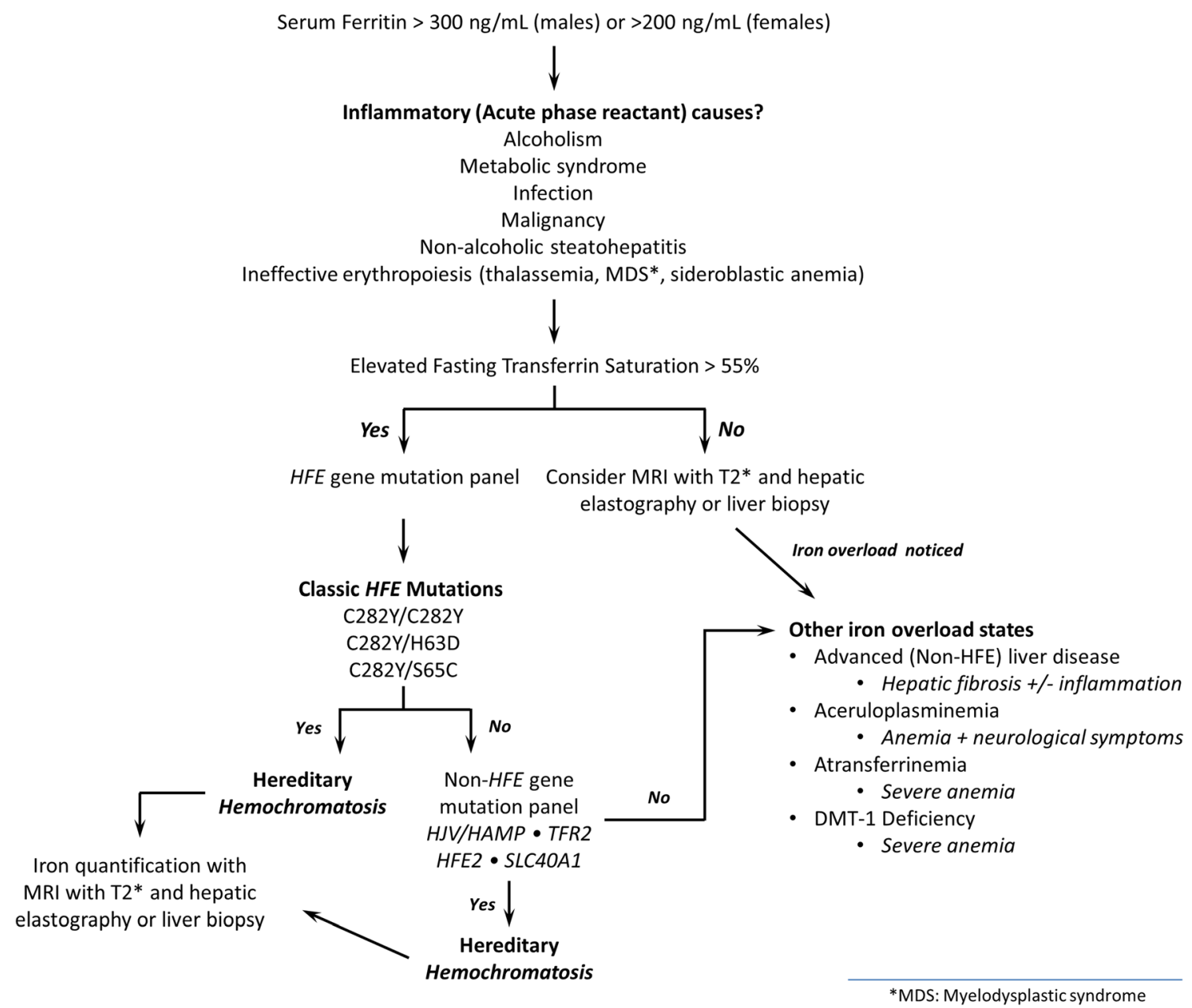

Figure 1 Diagnostic algorithm to iron overload state.

receptor (sTfR) is not an acute-phase reactant and the interpretation of iron status using sTfR measurement is not affected by these confounding pathologies. The concentration of sTfR in the blood can be a valuable indicator of iron status. ${ }^{22}$ We have found great utility for sTfR for differentiating an elevated ferritin caused by iron overload from those driven by systemic inflammation, and would recommend considering sTfR testing in select patients. STfR has also been proposed as a predictor of iron depletion induced by phlebotomy. ${ }^{23} H F E$ gene testing can be considered in patients with evidence of iron overload, and first-degree family members with iron overload or known HFE mutations. ${ }^{21}$

Those who have been found to have iron overload with a suspected genetic component can undergo non-HFE mutation panel testing. These gene panels typically assess for the four commonly reported non-HFE-associated genes outlined previously: HAMP, HFE2, SLC40A1, and TfR2, and are available from one of several commercially available labs. Figure 1 provides an algorithm used at our center for determining when to perform these non-HFE mutation panels.

\section{IMAGING}

MRI can qualitatively assess iron deposition in the liver, myocardium, spleen, pancreas, and heart via routinely performed sequences, in which affected tissues appear hypointense relative to normal on T2-weighted and in-phase T1-weighted images. This is independent of the underlying cause of the increased iron deposition, either primary $\mathrm{HH}$ or secondary hemosiderosis, although increased iron deposition in the pancreas strongly suggests the former. MRI can also more quantitatively estimate iron concentration in the liver and myocardium through the use of specific gradient-echo (GRE) sequences and their derived T2* ("T2 star") values. ${ }^{24}$ The heart can be more challenging to image due to cardiac motion, but this limitation can be overcome using GRE sequences gated to the electrocardiogram. There are published formulas for converting $\mathrm{T} 2 *$ values of both the liver and myocardium into tissue iron concentrations (in $\mathrm{mg} \mathrm{Fe} / \mathrm{g}$ dry tissue). ${ }^{25-27}$ The biggest limitation in estimating liver iron concentration with $\mathrm{T} 2 *$ is in the presence of superimposed hepatic steatosis, as the signal properties of fat and iron interfere with each other on GRE sequences. Fortunately, more recent sequences 
designed to independently measure both liver fat and iron can be used to overcome this limitation. There are some data to suggest that dual energy computed tomography (CT) may also be able to quantitatively measure iron concentration, ${ }^{28}$ but such techniques are not clinically widespread at this time.

MR, CT, and ultrasound imaging can provide surveillance for hepatic malignancy in hemochromatosis. Ultrasoundbased elastography (FibroScan) and its use in the spectrum of iron overload are poorly understood. A recent analysis of "technically limited/failed" MRI-based hepatic elastography studies in patients with iron overload found a $\mathrm{T} 2 *$ cutoff of $10 \mathrm{~ms}$ or lower on 1.5-T scanners as highly predictive of inadequate hepatic elastography values. ${ }^{29}$ These findings can potentially be translated to a recommendation to avoid relying on either MRI or ultrasound-based elastography values in patients with confirmed or high suspicion for significant hepatic iron infiltration.

MR elastography, initially developed as another GRE-based technique, has little benefit for quantification of fibrosis in significant hepatic iron overload as the susceptibility artifact present in iron-overloaded liver frequently renders the GREbased elastography sequences uninterpretable. It is unknown at this time that how much iron deposition must be present to render the elastography sequences unreliable; however, newer elastography sequences based on spin-echo techniques show promise in noninvasively quantifying liver fibrosis even in the setting of hepatic iron overload. ${ }^{30}$

\section{TREATMENT}

The cornerstone of treatment for iron overload is phlebotomy. ${ }^{31}$ Bi-weekly phlebotomy of $250-500 \mathrm{~cm}^{3}$ of blood for a hemoglobin (hgb) $>12.5 \mathrm{mg} / \mathrm{dL} /$ hematocrit (hct) $>$ $38 \%$ for men or a $\mathrm{hgb}>11.5 \mathrm{mg} / \mathrm{dL} / \mathrm{hct}>35 \%$ for women is indicated for induction of iron reduction with ferritin $>$ $1000 \mu / \mathrm{L}$. Weekly phlebotomy can be considered in otherwise healthy patients with a ferritin of $>1000 \mu / L$, if the frequency is tolerated. Patients with ferritin of $>1000 \mu / \mathrm{L}$ will typically require several months of induction phlebotomy to achieve iron depletion. If ferritin $<1000 \mu / \mathrm{L}$, monthly phlebotomy is reasonable. Goal ferritin is typically $50-100 \mu / \mathrm{L}^{1}$

Maintenance phlebotomies may need to be continued longterm, sometimes for life, and the frequency of maintenance therapy should be based on serum ferritin levels; most patients will be able to be managed with three to four maintenance phlebotomies annually. Chelation therapy, or removal of excess iron via medications, is rarely needed in genetic IOS. In patients with severe heart disease and/or anemia, the option for phlebotomy may be limited. Iron chelation with oral agents may be recommended in these circumstances. Iron chelators include desferrioxamine and deferasirox and are available as oral or intravenous formulation. Desferrioxamine is available as a subcutaneous formulation, or also via a battery-assisted infusion pump. Doses can be titrated based on 24-h urine iron concentrations. Deferasirox is a highly efficacious oral iron chelator that requires monitoring of renal function, but can provide adequate iron removal in certain patients. Significant side effects of all iron chelators should be considered (primarily GI complaints).

When hemochromatosis-related liver disease progresses to advanced-stage, despite iron reduction therapy, orthotropic liver transplantation may be the only viable therapeutic option. Surgical arthroplasty is considered if joint destruction becomes severe despite medical therapy.

\section{DIETARY CONSIDERATIONS}

Patients who have hereditary hemochromatosis should be advised to avoid iron supplementation. ${ }^{32}$ Ascorbic acid (vita$\min$ C) stimulates the absorption of iron, so excessive dietary intake of vitamin $\mathrm{C}$ or supplements should be discouraged. Alcohol use can promote disproportionate iron deposition in the liver and can also lead to a co-inflammatory steatohepatitis. Therefore, we recommend only limited alcohol use in noncirrhotics and complete alcohol abstinence in hemochromatosis patients with advanced fibrosis. Ingestion of raw shellfish is discouraged in all patients with cirrhotic liver disease from any cause, specifically due to the risk of Vibrio vulnificus infection.

Additional diet modifications such as coffee or tea consumption may help to reduce iron absorption. ${ }^{33}$ Limited red meat consumption, along with iron-fortified grains and cereals, can be considered. ${ }^{32}$ Heme iron (animal-derived) present in meat, fish, and poultry is more readily absorbed than nonheme (plant-based iron). While dietary changes can be useful in reducing the need for phlebotomy in the maintenance phase of phlebotomy, they will not eliminate the need for iron removal once overload is present.

\section{LONG-TERM MONITORING}

Patients with hemochromatosis need regular monitoring of hematocrit (hct), serum iron levels, and ferritin. Patients with cirrhosis have an estimated 20- to 200-fold increased risk of hepatocellular carcinoma as well as the risk of other complications of chronic liver disease (i.e., esophageal varices). Screening for hepatocellular with serum alpha-fetoprotein (AFP) every 6 months along with a liver ultrasonography is recommended. Patients with cirrhosis should be followed by a gastroenterologist/hepatologist.

Iron is known to be nephrotoxic at high levels. ${ }^{34}$ Acute renal failure in $\mathrm{HH}$ has been documented manifesting as crescentic glomerulonephritis and hemosiderin accumulation in the renal tubular epithelial cells. ${ }^{35}$ For patients with persistent iron overload, we recommend monitoring of renal function for signs of deterioration. Patients entering the 
maintenance stages of phlebotomy after appropriate iron removal are potentially at less risk for renal complications.

Cardiac infiltration of iron can lead to long-term risk of severe cardiomyopathy. Patients at risk for iron overload cardiomyopathy should be screened with echocardiogram every 1-2 years to identify abnormal ventricular diastolic function or decreased peak systolic tissue velocity. Those with higher iron content on cardiac MRI or cardiac biopsy should likely be screened more frequently. ${ }^{36}$

Endocrine dysfunction related to IOS may present as diabetes mellitus, or as a product of anterior pituitary infiltration. While long-term screening for endocrine dysfunction is not currently recommended, symptoms consistent with diabetes mellitus, hypogonadism, hypothyroidism, or adrenal dysfunction should prompt testing, and treatment where appropriate.

While certain HFE mutations may be associated with extrahepatic malignancy, no current adjusted screening guidelines exist for these patients.

\section{CONCLUSIONS}

Hereditary hemochromatosis is a relatively common genetic condition, especially people from certain key demographics. Correct and timely diagnosis along with appropriate treatment can nearly eliminate risk of long-term complications, which include multiple end-organ failure and liver cancer. Screening both symptomatic patients and certain asymptomatic persons can make a positive impact on long-term outcomes. Further study is needed to better define the risks associated with non$H F E$ hemochromatosis genetic profiles.

Corresponding Author: William C. Palmer, MD, FACP; Department of Gastroenterology and Hepatology Mayo Clinic, Jacksonville, FL, USA (e-mail: Palmer.william@mayo.edu).

\section{Compliance with Ethical Standards:}

Conflict of Interest: The authors declare that they do not have a conflict of interest.

\section{REFERENCES}

1. Bacon BR, Adams PC, Kowdley KV, Powell LW, Tavill AS, American Association for the Study of Liver D. Diagnosis and management of hemochromatosis: 2011 practice guideline by the American Association for the Study of Liver Diseases. Hepatology 2011;54:328-43.

2. Feder JN, Gnirke A, Thomas W, et al. A novel MHC class I-like gene is mutated in patients with hereditary haemochromatosis. Nat Genet 1996;13:399-408.

3. Pietrangelo A. Hereditary hemochromatosis: pathogenesis, diagnosis, and treatment. Gastroenterology 2010;139:393-408, e1-2.

4. Adams PC, Reboussin DM, Barton JC, et al. Hemochromatosis and iron-overload screening in a racially diverse population. N Engl J Med 2005;352: 1769-78

5. Cheng R, Barton JC, Morrison ED, et al. Differences in hepatic phenotype between hemochromatosis patients with HFE C282Y homozygosity and other HFE genotypes. J Clin Gastroenterol 2009;43:569-73.

6. Lv YF, Chang X, Hua RX, et al. The risk of new-onset cancer associated with HFE C282Y and H63D mutations: evidence from 87,028 participants. J Cell Mol Med 2016;20:1219-33.
7. Ruiz-Arguelles GJ, Garces-Eisele J, Reyes-Nunez V, et al. Heterozygosity for the H63D mutation in the hereditary hemochromatosis (HFE) gene may lead into severe iron overload in beta-thalassemia minor: observations in a thalassemic kindred. Rev Invest Clin 2001;53:117-20.

8. Terzi YK, Bulakbasi Balci T, Boga C, et al. Effect of Hereditary Hemochromatosis Gene (HFE) H63D and C282Y Mutations on Iron Overload in Sickle Cell Disease Patients. Turk J Haematol 2016.

9. Pietrangelo A. Non-HFE hemochromatosis. Hepatology 2004;39:21-9.

10. Lanktree MB, Sadikovic B, Waye JS, et al. Clinical evaluation of a hemochromatosis next-generation sequencing gene panel. Eur J Haematol 2017;98:228-34.

11. Badar S, Busti F, Ferrarini A, et al. Identification of novel mutations in hemochromatosis genes by targeted next generation sequencing in Italian patients with unexplained iron overload. Am J Hematol 2016;91:420-5.

12. Kew MC, Asare GA. Dietary iron overload in the African and hepatocellular carcinoma. Liver Int 2007;27:735-41.

13. Barton JC, Acton RT, Lee PL, West C. SLC40A1 Q248H allele frequencies and $\mathrm{Q} 248 \mathrm{H}$-associated risk of non-HFE iron overload in persons of sub-Saharan African descent. Blood Cells Mol Dis 2007;39:206-11.

14. Gordeuk VR, Caleffi A, Corradini E, et al. Iron overload in Africans and African-Americans and a common mutation in the SCLAOAl (ferroportin 1) gene. Blood Cells Mol Dis 2003;31:299-304.

15. Kumar S, Bandyopadhyay U. Free heme toxicity and its detoxification systems in human. Toxicol Lett 2005;157:175-88.

16. Robinson SR, Dang TN, Dringen R, Bishop GM. Hemin toxicity: a preventable source of brain damage following hemorrhagic stroke. Redox Rep 2009; 14:228-35.

17. Kroner PT, Koop A, Afsh M, Palmer WC. Hereditary hemochromatosis is associated with increased use of joint replacement surgery: results of a nationwide analysis. Digestive Disease Week 2018. Washington.2018.

18. Nagi S, Drissi C. Pituitary Hemochromatosis. In: MRI of the Pituitary Gland. Springer, Cham; 2016.

19. McNeil LW, McKee LC, Jr., Lorber D, Rabin D. The endocrine manifestations of hemochromatosis. Am J Med Sci 1983;285:7-13.

20. Osborne NJ, Gurrin LC, Allen KJ, et al. HFE C282Y homozygotes are at increased risk of breast and colorectal cancer. Hepatology 2010;51:1311-8.

21. Bacon BR. Screening for hemochromatosis. Arch Intern Med 2006; 166:269-70.

22. Khumalo H, Gomo ZA, Moyo VM, et al. Serum transferrin receptors are decreased in the presence of iron overload. Clin Chem 1998;44:40-4.

23. Pieroni L, Mekhloufi F, Thiolieres JM, Hainque B, Herson S, Jardel C. Soluble transferrin receptor in hemochromatosis patients during phlebotomy therapy. Clin Chim Acta 2005;353:61-6.

24. Anderson LJ, Holden S, Davis B, et al. Cardiovascular T2-star (T2*) magnetic resonance for the early diagnosis of myocardial iron overload. Eur Heart J 2001;22:2171-9.

25. Carpenter JP, He T, Kirk $\mathbf{P}$, et al. On T2* magnetic resonance and cardiac iron. Circulation 2011;123:1519-28.

26. Garbowski MW, Carpenter JP, Smith G, et al. Biopsy-based calibration of T2* magnetic resonance for estimation of liver iron concentration and comparison with R2 Ferriscan. J Cardiovasc Magn Reson 2014;16:40.

27. Storey P, Thompson AA, Carqueville CL, Wood JC, de Freitas RA, Rigsby CK. R2* imaging of transfusional iron burden at $3 \mathrm{~T}$ and comparison with 1.5T. J Magn Reson Imaging 2007;25:540-7.

28. Luo XF, Xie XQ , Cheng $\mathbf{S}$, et al. Dual-Energy CT for Patients Suspected of Having Liver Iron Overload: Can Virtual Iron Content Imaging Accurately Quantify Liver Iron Content? Radiology 2015;277:95-103.

29. Kroner PT, Stancampiano FF, Bowman A, Vishnu P, Heckman MG, Diehl NN, McLeod E, Nikpour N, Palmer wC. Hepatic iron overload identified by magnetic resonance imaging-based $\mathrm{T} 2 *$ is a predictor of inadequate hepatic elastography Digestive Disease Week 2018. Washington, D.C; 2018.

30. Mariappan YK, Dzyubak B, Glaser KJ, et al. Application of Modified Spin-Echo-based Sequences for Hepatic MR Elastography: Evaluation, Comparison with the Conventional Gradient-Echo Sequence, and Preliminary Clinical Experience. Radiology 2017;282:390-8.

31. Niederau C, Fischer R, Purschel A, Stremmel W, Haussinger D, Strohmeyer G. Long-term survival in patients with hereditary hemochromatosis. Gastroenterology 1996;110:1107-19.

32. https://www.niddk.nih.gov/health-information/health-topics/liver-disease/hemochromatosis/Pages/facts.aspx\#eating. Date accessed: 11/8/ 2017.

33. Morck TA, Lynch SR, Cook JD. Inhibition of food iron absorption by coffee. Am J Clin Nutr 1983;37:416-20. 
34. Sponsel HT, Alfrey AC, Hammond WS, Durr JA, Ray C, Anderson RJ Effect of iron on renal tubular epithelial cells. Kidney Int 1996;50:436-44.

35. Ozkurt S, Acikalin MF, Temiz G, Akay OM, Soydan M. Renal hemosiderosis and rapidly progressive glomerulonephritis associated with primary hemochromatosis. Ren Fail 2014;36:814-6.
Gujja P, Rosing DR, Tripodi DJ, Shizukuda Y. Iron overload cardiomyopathy: better understanding of an increasing disorder. J Am Coll Cardiol 2010;56:1001-12. 